\title{
Infinite-Dilution Viscoelastic Properties of Star Polystyrene with Nine Arms
}

\author{
Yutaka Mitsuda, Kunihiro Osaki, John \\ L. SChrag, and John D. FERrY \\ Department of Chemistry and Rheology Research \\ Center, University of Wisconsin, Madison, \\ Wisconsin 53706, U.S. A.
}

(Received March 6, 1972)

\begin{abstract}
The storage and loss shear moduli, $G^{\prime}$ and $G^{\prime \prime}$, have been measured for solutions of a star polystyrene with average number of arms 8.7 and weight-average molecular weight 5000000 , by use of the Birnboim-Schrag multiple-lumped resonator. The frequency range was 106 to $6060 \mathrm{~Hz}$ and the concentration range 0.001 to $0.013 \mathrm{~g}$ / $\mathrm{m} l$. Decalin and di-2-ethyl hexyl phthalate were used as solvents at their respective $\Theta$ temperatures, 15 and $21^{\circ} \mathrm{C}$, and $\alpha$-chloronaphthalene as a good solvent at $25^{\circ} \mathrm{C}$. The extrapolated intrinsic moduli agree well with the predictions of the Zimm-Kilb theory as evaluated by Osaki and Schrag if the hydrodynamic interaction parameter $h^{*}$ is taken as 0.40 in the $\Theta$ solvents and 0.25 in $\alpha$-chloronaphthalene. The product $h^{*} \alpha_{\eta}$, where $\alpha_{\eta}$ is the expansion factor of the excluded-volume effect as evaluated from the intrinsic viscosity, is about 0.40 instead of 0.21 as observed for linear polymers. The origin of this unexpectedly large value is unclear. The terminal relaxation time is smaller by a factor of 12 to 14 than that of a linear polymer of the same molecular weight; the storage modulus at a given low frequency and concentration, and the steady-state compliance, are correspondingly much smaller than for a linear polymer.

KEY WORDS Viscoelasticity / Dilute Solutions / Multiple-lumped

Resonator / Intrinsic Modulus / Star-Shaped Polymer / Polystyrene /

Zimm-Kilb Theory /
\end{abstract}

Several recent studies of viscoelastic properties of linear polymers in solutions sufficiently dilute to permit extrapolation to infinite dilution have provided quantitative comparisons with molecular theories of polymer dynamics. Measurements in theta solvents confirmed the theory of Zimm; ${ }^{1}$ those in good solvents were compared first $^{2,3}$ with the Tschoegl ${ }^{4}$ modification of the theory of Zimm, and subsequently ${ }^{5,6}$ with an evaluation of the Zimm theory by an exact eigenvalue solution, due to Lodge and $\mathrm{Wu}^{7}$, taking varying hydrodynamic interaction into account.

We are now undertaking a study of dilute solutions of polymers with various types of branched structures. Their viscoelastic properties, extrapolated to infinite dilution, reveal distinctive features of frequency dependence, hydrodynamic interaction, and viscoelastic constants such as steady-state compliance. The behavior of a 4-arm star polybutadiene in a good solvent has been reported. ${ }^{5}$ The present paper describes a 9-arm star polystyrene in both good and theta solvents.

\section{EXPERIMENTAL}

\section{Materials}

The star polystyrene was generously furnished by Drs. D. J. Worsfold and J.-G. Zilliox. It was prepared at the Centre de Recherches sur les Macromolecules, Strasbourg, by anionic block copolymerization of a linear polystyrene precursor with a very small amount of divinyl benzene. $^{8}$ This sample (M5E) had a weightaverage molecular weight (M) of 5000000 , with an arm (precursor) molecular weight of 573000 , corresponding to an average number of arms of 8.7. In subsequent calculations, this will be taken as 9. It is believed to have a very sharp molecular weight distribution. From 
measurements of light scattering and intrinsic viscosity, ${ }^{9}$ the ratio of square of radius of gyration to the corresponding value for a linear polymer of the same molecular weight in a $\Theta$ solvent was found to be $g=0.311$ (in good agreement with the theoretical value from the equation of Orofino ${ }^{10}$ ) and the analogous ratio of intrinsic viscosities in a theta solvent was found to be $g^{\prime}=0.451$. The intrinsic viscosity in cyclohexane at the $\Theta$ temperature was 76.5 $\mathrm{m} l / \mathrm{g}$.

The solvents were $\alpha$-chloronaphthalene (superior), Decalin (approximately half cis and half trans, practical), and di-2-ethyl hexyl phthalate ("DOP", practical), all from Matheson, Coleman, and Bell. Their viscosities at the temperatures of viscoelastic measurements were, respectively, 0.0300 at $25^{\circ} \mathrm{C}, 0.0303$ at $15^{\circ} \mathrm{C}$, and 0.732 poise at $21^{\circ} \mathrm{C}$. The latter two temperatures were chosen to approximate the $\Theta$ points in Decalin and DOP respectively; the $\Theta$ temperatures for linear polystyrene ${ }^{3}$ are 16 and $22^{\circ} \mathrm{C}$, and according to Zilliox ${ }^{9}$ they should be slightly lower for the branched polymer.

Solutions were made up with very gentle stirring at room temperature. The most concentrated solution was measured first and then sequentially diluted to several lower concentrations.

\section{Method}

The storage and loss shear moduli, $G^{\prime}$ and $G^{\prime \prime}$, of the solutions were measured with the Birnboim-Schrag multiple-lumped resonator with computerized data acquisition and processing system. ${ }^{11}$ The use of two resonators gave 9 working resonance frequencies from 100 to $6060 \mathrm{~Hz}$. The concentration ranges $(c)$ were 0.0011 to $0.0036 \mathrm{~g} / \mathrm{m} l$ in 8 steps in $\alpha$-chloronaphthalene, 0.0044 to 0.013 in 9 steps in Decalin, and 0.0016 to 0.0107 in 6 steps in DOP. Detailed numerical data will be given elsewhere. ${ }^{12}$

\section{RESULTS AND DISCUSSION}

\section{Concentration Dependence and Extrapolation}

The quantities $\left(G^{\prime} / c\right)^{1 / 2}$ and $\left(G^{\prime \prime}-\omega \eta_{\mathrm{s}}\right) / c$, where $\omega$ is radian frequency and $\eta_{\mathrm{s}}$ is solvent viscosity, were plotted against $c$ and extrapolated to zero $c$ at each frequency as described in previous studies $^{3,5,6}$ to give the corresponding intrinsic quantities $\left[G^{\prime}\right]$ and $\left[G^{\prime \prime}\right]$. The initial concentration dependences may be described by the ratios $\gamma^{\prime}=\left(G^{\prime} / c\right)^{-1} \mathrm{~d}\left(G^{\prime} / c\right) / \mathrm{d} c$ and $\gamma^{\prime \prime}$ which is the corresponding expression with $G^{\prime \prime}-\omega \eta_{\mathrm{s}}$ substituted for $G^{\prime}$. Several values of $\gamma^{\prime}$ and $\gamma^{\prime \prime}$, together with their dimensionless ratio $\gamma$, are listed in Table I.

At low frequencies, $\gamma^{\prime \prime}$ is equivalent to $k^{\prime}[\eta]$, where $k^{\prime}$ is the familiar Huggins constant; the

Table I. Parameters describing initial concentration dependence

\begin{tabular}{lrrrr}
\hline \multicolumn{1}{c}{ Solvent } & Frequency, & \multicolumn{1}{c}{$\gamma^{\prime}$} & $\gamma^{\prime \prime}$ & $\gamma$ \\
\hline$\alpha$-Chloronaphthalene & 106 & 600 & 140 & 4.4 \\
& 420 & 270 & 62 & 2.3 \\
\multirow{3}{*}{ Decalin } & 2520 & 52 & 14 & 1.9 \\
& 540 & 330 & 44 & 5.6 \\
& 1400 & 140 & 31 & 4.4 \\
& 2520 & 82 & 20 & 4.1 \\
& 3430 & 75 & 17 & 4.4 \\
\hline
\end{tabular}

values in Table I correspond to $k^{\prime}=0.49$ in $\alpha$ chloronaphthalene and 0.50 in Decalin, which are reasonable. The fact that both $\gamma^{\prime}$ and $\gamma^{\prime \prime}$ decrease rapidly with increasing frequency is consistent with the hypothesis that the longest relaxation time is much more strongly concentration-dependent at very low concentrations than are the other relaxation times, according to an earlier phenomenological treatment ${ }^{3}$ in which the initial effect of concentration was supposed to consist of slight increases in relaxation times with no alteration of the relaxation strength (all contributions to modulus proportional to $c R T / M)$. Following this treatment, the relative changes of different relaxation times with concentration can also be examined in terms of the values of $\gamma$ at the lowest frequencies; if only the longest relaxation time is affected at first, $\gamma$ should be $2 S_{1} / S_{2}$ where $S_{1}=\sum_{p}\left(\tau_{p} / \tau_{1}\right)$ and $S_{2}=\sum_{p}\left(\tau_{p} / \tau_{1}\right)^{2}, \tau_{p}$ being the relaxation times corresponding to motional modes of the macromolecule. ${ }^{3}$ With the values of $S_{1}$ and $S_{2}$ correponding to the theoretical fit to the frequency dependence described below, $\gamma$ should be 3.1 in $\alpha$-chloronaphthalene and 3.4 in Decalin at low frequencies, whereas for equal concentration 
dependence of all relaxation times, $\gamma=2$. Since the observed values of $\gamma$ are higher than any of these, the initial concentration dependence of star polymers may require a more elaborate description in which changes in relaxation strength are taken into account. However, the observed $\gamma$ seems clearly inconsistent with equal concentration dependence of all relaxation times.

\section{Extrapolated Viscoelastic Functions}

The extrapolated values $\left[G^{\prime}\right]$ and $\left[G^{\prime \prime}\right]$ are reduced to $\left[G^{\prime}\right]_{\mathrm{R}}=\left[G^{\prime}\right] M / R T$ and $\left[G^{\prime \prime}\right]_{\mathrm{R}}=\left[G^{\prime \prime}\right] M / R T$ and then plotted logarithmically against $\omega \tau_{01} S_{1}$

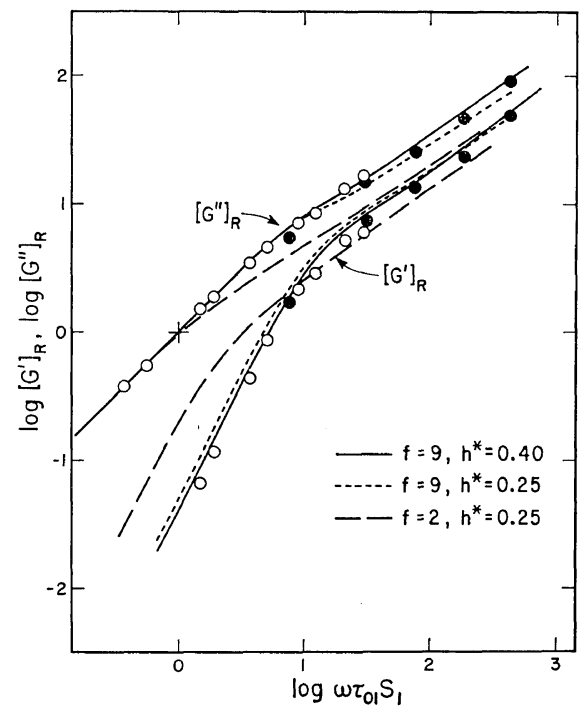

Figure 1. Logarithmic plots of reduced intrinsic shear moduli $\left[G^{\prime}\right]_{\mathrm{R}}$ and $\left[G^{\prime \prime}\right]_{\mathrm{R}}$ against $\omega \tau_{01} S_{1}$ for two $\Theta$ solvents: open circles, Decalin; black circles, DOP. Data in DOP shifted by $\log (288 / 294)=$ -0.01 on both axes to reduce to $\Theta$-temperature of Decalin. Curves: exact eigenvalue evaluation of Zimm-Kilb theory with $f=9, N_{\mathrm{b}}=100, h^{*}=0.40$ and 0.25 as identified respectively as shown; also exact eigenvalue evaluation of Zimm theory for linear molecules, $f=2, N=100, h^{*}=0.25$.

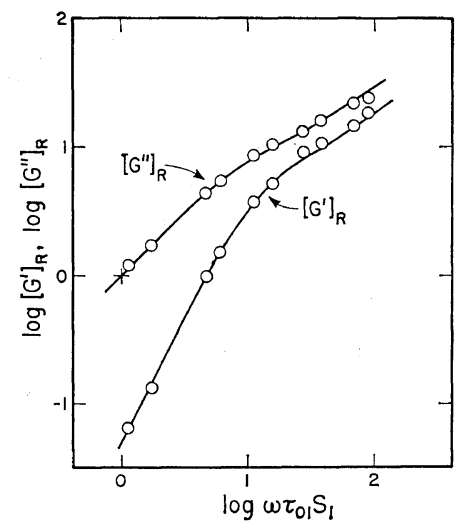

Figure 2. Logarithmic plots of $\left[G^{\prime}\right]_{R}$ and $\left[G^{\prime \prime}\right]_{R}$ against $\omega_{\tau 01} S_{1}$ for $\alpha$-chloronaphthalene. Curves: exact eigenvalue evaluation of Zimm-Kilb theory with $f=9, N_{\mathrm{b}}=100, h^{*}=0.25$.

in Figures 1 and 2. Here $\tau_{01}$ is the terminal relaxation time at infinite dilution, calculated ${ }^{13}$ as $\tau_{01}=\eta_{\mathrm{s}}[\eta] M / R T S_{1}$. The intrinsic viscosities were obtained both from conventional capillary viscometry and (except for DOP, for which MLR measurements did not extend to low enough frequency) from $\left[G^{\prime \prime}\right]$ at low frequencies by the relation $[\eta]=\lim _{\omega \rightarrow 0}\left[G^{\prime \prime}\right] / \omega \eta_{\mathrm{s}}$; these sources were in good agreement, as shown in Table II, and the values in the $\theta$ solvents agreed reasonably well with Zilliox's result in cyclohexane. The sum $S_{1}$ is based on the fit to the Zimm-Kilb theory as described below; it is 14.15 in the $\theta$ solvents and 12.96 in $\alpha$-chloronaphthalene. $\log$ $\tau_{01}$ is also listed in Table II.

To combine the data in the two $\theta$-solvents, the ordinates and abscissas of the DOP points were very slightly adjusted by $\log (288 / 294)$ to reduce them to the $\Theta$-temperature of Decalin. The two sets of data combine well to give single curves, confirming once again ${ }^{3,5}$ the proportionality of relaxation times to solvent viscosity.

Table II. Intrinsic viscosities and terminal relaxation times

\begin{tabular}{lcccccc}
\hline \multicolumn{1}{c}{ Solvent } & Temp, ${ }^{\circ} \mathrm{C}$ & {$[\eta]^{\mathrm{a}}, \mathrm{m} l / \mathrm{g}$} & {$[\eta]^{\mathrm{b}}, \mathrm{m} l / \mathrm{g}$} & {$[\eta] /[\eta]_{1}$} & $\log \tau_{01}, \mathrm{sec}$ & $\tau_{01} / \tau_{011}$ \\
\hline Decalin & $15^{\circ}$ & 85 & 88 & 0.48 & -4.40 & 0.081 \\
DOP & $21^{\circ}$ & - & 80 & - & - & - \\
$\alpha$-Chloronaphthalene & $25^{\circ}$ & 290 & 283 & 0.43 & -3.88 & 0.073 \\
\hline
\end{tabular}

\footnotetext{
a From multiple-lumped resonator at low frequency.

b From capillary measurements.
} 
The frequency dependence of viscoelastic properties of star-shaped macromolecules can be calculated on the basis of the familiar beadspring model by the theory of Zimm and Kilb. ${ }^{14}$ This has been numerically evaluated with exact eigenvalues by Osaki and Schrag, ${ }^{15}$ for various combinations of numbers of arms $(f)$, submolecules per arm $\left(N_{\mathrm{b}}\right)$, and hydrodynamic interaction parameter $\left(h^{*}\right)$. The results are not very sensitive to $N_{\mathrm{b}}$ if this parameter is sufficiently large. Taking $f=9$ and $N_{\mathrm{b}}=100$, theoretical logarithmic curves for $\left[G^{\prime}\right]_{\mathrm{R}}$ and $\left[G^{\prime \prime}\right]_{\mathrm{R}}$ against $\omega \tau_{01}$ were drawn for various values of $h^{*}$ and the best match to the experimental points was obtained. The procedure involves matching the shape of the experimental plot using $\log \omega \eta_{\mathrm{s}}[\eta] M / R T\left(=\log \omega \tau_{01} S_{1}\right)$ as abscissa while shifting the origin of the theoretical curve by $\log S_{1}$; both $S_{1}$ and the shape depend on the selection of $h^{*}$, but the $\log S_{1}$ shift is automatically achieved by coincidence of $\left[G^{\prime \prime}\right]_{R}$ at low frequencies. The ordinate scale is, of course, fixed.

In the $\Theta$ solvents, a fairly good fit is obtained for $h^{*}=0.25$ (Figure 1), but $h^{*}=0.40$ appears to be distinctly better especially at low frequencies. The latter value is surprisingly high $\operatorname{since}{ }^{15} h^{*}=$ 0.25 corresponds to the dominant hydrodynamic interaction of the Zimm-Kilb theory and the physical significance of higher values is uncertain except that they seem to imply a "super-non-free-draining". For $h^{*}=0.40$ and $f=9, S_{1}=14.15$ and $S_{2}=8.41$. Theoretical curves fo ra linear polymer $(f=2)$ with the same molecular weight in a $\Theta$ solvent $\left(h^{*}=0.25\right)$ are shown for comparison. As previously noted for a 4arm star, the branched polymer has much smaller $G^{\prime}$ at low frequencies and its frequency dependence shows characteristic inflections or "bumps".

In $\alpha$-chloronaphthalene, a good fit is obtained with $h^{*}=0.25 ; S_{1}=12.96$ and $S_{2}=8.37$. It is of interest that the product $h^{*} \alpha_{\eta}$ (where $\alpha_{\eta}^{3}$ is the ratio of intrinsic viscosities in $\alpha$-chloronaphthalene and $\Theta$-solvents) is about 0.38 ; so the approximate constancy of this product observed for linear polymers ${ }^{6}$ holds also for the 9-arm star, except that the product is about 0.40 instead of 0.21 as found for linear polymers.

The abnormally high value of $h^{*}$ is probably associated with the fact that $\log g^{\prime} / \log g$ is 0.68 instead of $1 / 2$ as predicted by the Zimm-Kilb theory, also that $g^{\prime}=0.451$ is smaller than given by the numerical evaluation of the Zimm-Kilb theory, about 0.7 for $f=9$ and $N_{b}=100$. For a 4-arm star polybutadiene, $h^{*}$ was found ${ }^{5}$ to be slightly smaller $(0.10)$ than for the linear polymer $(0.15)$ in the same good solvent. It is possible that at low degrees of branching, $h^{*}$ decreases with increasing $f$ because the chain dimensions are somewhat extended; the latter effect is evident from both light scattering and intrinsic viscosity ${ }^{9,16}$ especially for stars with shorter branches, and the inverse proportionality of $h^{*}$ to $\alpha_{\eta}$ observed for linear polymers, where $\alpha_{\eta}^{3}$ is the ratio of intrinsic viscosities in good and theta solvents, suggests that $h^{*}$ should decrease in consequence. However, $h^{*}$ is proportional to the friction coefficient $\zeta$ which measures the frictional resistance of a bead (in the bead-spring model of the Zimm or ZimmKilb theory) for translation through its environment. For still higher values of $f$, an increase in $\zeta$ associated with an abnormally large density of polymer segments in the interior of the molecule may cause the effective value of $h^{*}$ to increase as observed. Clearly the Zimm-Kilb theory requires modification to take these effects into account.

\section{Comparison of Branched and Linear Polymers}

The intrinsic viscosity $[\eta]$ and terminal relaxation time $\tau_{01}$ of a linear polymer with the same molecular weight in a $\Theta$ solvent are readily calculated from earlier data on linear polymers, ${ }^{3}$ and also in $\alpha$-chloronaphthalene if the Mark-Houwink coefficient for intrinsic viscosity is assumed to be 0.68 . The ratios of these quantities, branched to linear, $[\eta] /[\eta]_{1}$ and $\tau_{01} / \tau_{011}$, are included in Table II. It is striking that, whereas the intrinsic viscosity of the branched polymer is smaller by a factor of 2.1 to 2.3 , the terminal relaxation time is smaller by a factor of 12 to 14 . The small values of $\tau_{01}$ are associated with the large values of $S_{1}$ and $S_{2}$ cited above and these in turn reflect the degenerate nature of the normal modes of the Zimm-Kilb theory. That this degenerate description is essentially correct is indicated by the excellent agreement with theory. 
The small terminal relaxation time is also reflected in a very much smaller value of $G^{\prime}$ at a given low frequency and low concentration, and a very much smaller steady-state compliance. These features should make it possible to detect and estimate the extent of branching from dilute solution measurements. However, a practical problem remains since the existence of a broad molecular weight distribution will affect the steady-state compliance in the opposite direction.

Acknowledgment. This work was supported in part by grants from the Army Research Office (Durham), the National Science Foundation, and the National Institutes of Health.

\section{REFERENCES}

1. B. H. Zimm, J. Chem. Phys., 24, 269 (1956).

2. (a) H. Tanaka, A. Sakanishi, and J. Furuichi, J. Polym. Sci., Part C, 15, 317 (1966); (b) A. Sakanishi and H. Tanaka, "Proceedings of 5th International Congress on Rheology," Vol. 4, S. Onogi, Ed., Tokyo Univ. Press, Tokyo, 1970, pp 251 and 259.

3. R. M. Johnson, J. L. Schrag, and J. D. Ferry,
Polymer J. (Japan), 1, 742 (1970).

4. N. W. Tschoegl, J. Chem. Phys., 39, 149 (1963); 40, 473 (1964).

5. K. Osaki, Y. Mitsuda, R. M. Johnson, J. L. Schrag, and J. D. Ferry, Macromolecules, 5, 17 (1972).

6. K. Osaki, J. L. Schrag, and J. D. Ferry, Macromolecules, 5, 144 (1972).

7. A.S. Lodge and Y. J. Wu, to be published.

8. D. J. Worsfold, J.-G. Zilliox, and P. Rempp, Can. J. Chem., 47, 3379 (1969).

9. J.-G. Zilliox, personal communication.

10. T. A. Orofino, Polymer Preprints, 9, 260 (1968).

11. J. L. Schrag and R. M. Johnson, Rev. Sci. Instr., 42, 224 (1971).

12. Y. Mitsuda, forthcoming thesis, University of Wisconsin.

13. J. D. Ferry, "Viscoelastic Properties of Polymers," 2nd ed., Wiley, New York, N. Y., 1970, p 221.

14. B. H. Zimm and R. W. Kilb, J. Polym. Sci., 37, 19 (1959).

15. K. Osaki and J. L. Schrag, J. Polym. Sci., submitted.

16. I. Noda, T. Horikawa, T. Kato, T. Fujimoto, and M. Nagasawa, Macromolecules, 3, 795 (1970). 